\title{
EDITORIALS
}

\section{The debated problem of community-acquired pneumonia diagnosis: many guidelines, any guideline?}

\author{
*Antonio Infantino1, Ruben Infantino² \\ 1 Respiratory Area, Società Italiana Interdisciplinare per le Cure \\ Primarie (SIICP), Italy \\ 2 Respiratory Diseases Institute, University of Bari, Italy \\ *Correspondence: Dr. Antonio Infantino, SIICP - Società Italiana \\ Interdisciplinare per le Cure Primarie, Vice-President and Chair \\ (in charge) of the Respiratory Area, GP in Casamassima Surgery \\ at Corso G. Garibaldi 17/A 70010 Casamassima (BA), Italy. \\ Tel: +393396356582 Fax: +3908067 7001 \\ E-mail: antonio.infantino.md@gmail.com
}

See linked articles by Snijders et al. on pg 400 and

Christensen et al. on pg 454

The term community-acquired pneumonia (CAP) refers to a common lower respiratory infection diagnosed by a combination of some or all of the following: clinical signs and symptoms, an infiltrate seen on chest radiography, and abnormal laboratory values. It occurs outside of the hospital, or within 48 hours after hospital admission in a patient who has not been recently hospitalised and is not living in a long-term care facility. Pneumonia acquired in hospital or while living in an inpatient setting is referred to as "nosocomial pneumonia". The clinical symptoms and signs of CAP include cough (with or without sputum production), fever, chills, tachypnoea, tachycardia, pleuritic chest pain, dyspnoea, altered mental status, dehydration, and hemoptysis; clinical findings will include a temperature greater than $37.8^{\circ} \mathrm{C}$, heart rate over $100 / \mathrm{min}$, respiratory rate greater than $25 / \mathrm{min}$, oxygen saturations in room air $<90 \%$, rhonchi or focal rales on auscultation of the lungs, decreased breath sounds, and bronchophony.

CAP is a major health problem worldwide and is associated with considerable morbidity, mortality and health care costs. However, although data are available from many prospective studies and national databases, it is difficult to determine the real clinical and economic impact of CAP for a number of reasons, the most common being that diagnostic certainty is usually only obtained in the hospital setting where (unlike the primary care setting) all diagnostic tools are readily available.

Therefore, to determine the burden of CAP affecting adults in North America and in Europe, two comprehensive literature reviews were conducted recently to examine the incidence, morbidity and mortality, aetiology, antibiotic resistance, and economic impact of the disease in these populations. ${ }^{1.2}$ Both in the US and Europe, CAP is the most frequent cause of infection-related death. Its estimated incidence varies between countries and is dependent on age and gender, being higher in individuals aged $\geq 65$ years and in men; therefore the incidence is expected to increase further as the average age of the population in the US and Europe increases. CAP is the fifth to ninth leading cause of death in developed countries, mainly among young children and elderly people, with mortality rates varying between $6.4 \%$ to $40 \%$ depending on the care setting (e.g. home, hospital, intensive care unit). ${ }^{2}$ CAP accounts for more than $\$ 17$ billion annually in the United States.' In Europe, pneumonia costs nearly $€ 10.1$ billion annually, with inpatient care accounting for $€ 5.7$ billion, outpatient care $€ 0.5$ billion, drugs $€ 0.2$ billion and the indirect cost of lost work days amounts to $€ 3.6$ billion. ${ }^{2}$ CAP also has considerable effects on quality of life, with the time taken for patients to return to full activity ranging from 7 to 43 days.

Both in the US and Europe, Streptococcus pneumoniae continues to be the most frequently identified pathogen associated with CAP. Resistance to antibiotics is seen in all pathogens associated with CAP and is similar on both continents. ${ }^{1,2}$ Strep. pneumoniae resistance to penicillin and macrolides is increasing, but whether this correlates with increased mortality is still uncertain. ${ }^{3}$ Failure of first line antibiotic therapy is one of the causes of increasing treatment costs. ${ }^{3}$

The hospital admission rates for CAP vary between different studies, but on average are fairly low at about $25-30 \% .^{1,2,4}$ Therefore, primary care clinicians have a key role in the diagnosis and management of these patients..$^{5}$ This key role has been absolutely reaffirmed by the study from Snijders et al. ${ }^{6}$ published in this issue of the PCRJ. This interesting paper from the Netherlands is notable for the size of the study population (nearly 400,000 ) and the thoroughness of the research method. Between 2002 and 2009, the authors were able to study approximately 3,700 CAP episodes per year, of which $79 \%$ were managed solely in the primary care setting, showing clearly the contribution made by general practitioners (GPs) to the management of CAP.

The study from Snijders et al. ${ }^{6}$ does have some limitations, the main one being (as declared by the authors) that the GP diagnosis of CAP was based on clinical symptoms and mostly not confirmed by the presence of infiltrate abnormalities on the chest $x$-ray or any laboratory findings. This highlights once again the ongoing debate regarding CAP diagnosis, and whether the GPs were seeing CAP or 'suspected CAP', a difference of not inconsiderable importance when trying to determine the real burden of the illness and the management strategies employed by national health services. 
This debate is further highlighted by another study in this issue from Christensen et al. ${ }^{7}$ Using a cross-sectional design, the authors investigated, at primary care level, the prevalence of presumed pneumonia in 2,698 patients with lower respiratory tract infection (LTRI) in two countries (Denmark and Spain) with different antibiotic prescribing rates. $47 \%$ of patients in Denmark were classified as having pneumonia, whereas in Spain this figure was 11\%. In Spain, fever and a positive x-ray weighted significantly more in the diagnosis of pneumonia than in Denmark. Danish GPs, conversely, attached more importance to dyspnoea/polypnoea and CRP tests.

How accurate is an empiric diagnosis of CAP based on clinical assessment alone? A recent study of CAP diagnosis in 2,810 patients from 12 European countries shows that the majority of diagnoses of radiographic pneumonias were not suspected on clinical grounds by GPs. ${ }^{8}$ In the primary care setting, and despite rigorous definitions of pneumonia which require the finding of a pulmonary infiltrate on a chest radiograph, ${ }^{9}$ in patients with mild respiratory symptoms a chest radiograph will often not be ordered. This scenario is complicated further by the possibility that patients with bacterial "atypical" pneumonia can have a normal white cell count ${ }^{10}$ (assuming a blood count is requested), few physical findings, and even negative chest $\mathrm{x}$-rays in patients with dehydration or in the early stage of the disease.

Few population-based studies of CAP treated in the primary care setting have been undertaken. In a meta-analysis of the prognosis and outcomes of CAP, only six of the 127 studies included in the review were carried out in ambulatory cohorts with radiographic confirmation, and one of the conclusions was that future investigations should focus greater attention on studying ambulatory patients. ${ }^{11}$ Despite the fact that primary care plays an important role in the management of CAP, as confirmed by Snijders et al., ${ }^{6}$ the potentially low diagnostic yield (20\% of cases) at this level is surprising; this may be explained by the lack of availability of chest radiography so that many patients are referred to the hospital emergency department, as well as the tendency of patients to seek care directly from the hospital emergency service. ${ }^{11}$

Since 1993, a considerable number of guidelines for the

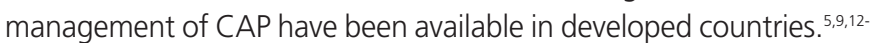
${ }^{15}$ However, with one or two key exceptions, ${ }^{5,12}$ these seem to be notable for their lack of relevance and friendliness to primary care. The guideline process, which first began in the US and Canada, has been implemented over time in numerous countries throughout the world, and now each geographic region or country has developed locally specific recommendations. The result is a lack of homogeneity between guidelines (e.g. are x-rays mandatory or not?) and actual contradictions (should we reserve the definition of CAP only to those LRTIs confirmed by chest $\mathrm{x}$-ray, and have a diagnosis of 'suspcted $C A P^{\prime}$ for those diagnosed on clinical features alone?). It is also interesting to note that guidelines from different regions often interpret the same evidence base differently even though the bacteriology of CAP varies little from one region to another. ${ }^{4}$

However, the question remains: is it necessary to review the global consensus on CAP to improve our approach in order to reduce the comprehensive burden of the disease? Snijders and colleagues $^{6}$ have confirmed that GPs play a crucial role in the management of this condition. Christensen et al. ${ }^{7}$ have confirmed different diagnostic approaches in different countries. In the interim, it is good practice to maximise the numbers of patients having vaccination against flu virus and pneumococcal pneumonia.

Conflicts of interest The authors declare that they have no conflicts of interest in relation to this article.

Commissioned article; not externally peer-reviewed; accepted 12th November 2013; online 23rd November 2013

C 2013 Primary Care Respiratory Society UK. All rights reserved

http://dx.doi.org/10.4104/pcrj.2013.00096

Prim Care Respir J 2013; 22(4): 383-385

\section{References}

1. File TM Jr, Marrie TJ. Burden of community-acquired pneumonia in North American adults. Postgrad Med 2010;122(2):130-41 http://dx.doi.org/10.3810/pgm.2010.03.2130

2. Welte T, Torres A, Nathwani D. Clinical and economic burden of communityacquired pneumonia among adults in Europe. Thorax 2012;67(1):71-9. http://dx.doi.org/10.1136/thx.2009.129502. Epub 2010 Aug 20.

3. Blasi F, Centanni S, Rossi A. Vaccinazione antipneumococcica nell'adulto. L'impatto clinico della patologia pneumococcica nell'adulto. Medicina Toracica. Anno XXXII, Supplemento 2, 2012

4. Niederman MS, Luna CM. Community-acquired pneumonia guidelines: a global perspective. Seminars in respiratory and critical care medicine 2012;33(3):298-310. http://dx.doi.org/10.1055/s-0032-1315642 Epub 2012 Jun 20.

5. Levy ML, Le Jeune I., Woodhead MA, Macfarlane JT, Lim WS. Primary care summary of the British Thoracic Society Guidelines for the management of community acquired pneumonia in adults: 2009 update. Endorsed by the Royal College of General Practitioners and the Primary Care Respiratory Society UK. Prim Care Respir J 2010;19(1):21-7. http://dx.doi.org/10.4104/pcrj.2010.00014

6. Snijders BEP, van der Hoek W, Stirbu I, van der Sande MAB, van Gageldonk-Lafeber $A B$. General practitioners' contribution to the management of community acquired pneumonia in the Netherlands: a retrospective analysis of primary care, hospital, and national mortality databases with individual data linkage. Prim Care Respir $J$ 2013;22(4):400-05. http://dx.doi.org/10.4104/pcrj.2013.00085

7. Christensen SF, Jørgensen LC, Cordoba G, Llor C, Siersma V, Bjerrum L. Marked differences in GPs' diagnosis of pneumonia between Denmark and Spain: a crosssectional study. Prim Care Respir J 2013; 22(4):454-8. http://dx.doi.org/10.4104/pcrj.2013.00093

8. Vugt SF, Verheij T, Jong PD, et al. on behalf of the GRACE Project Group (www.grace-Irti.org). Diagnosing pneumonia in patients with acute cough: clinical judgement compared to chest radiography. Eur Resp J 2013;42(4):1076-82. http://dx.doi.org/10.1183/09031936.00111012. Epub 2013 Jan 24

9. Mandell LA, Wunderink RG, Anzueto A, et al. Infectious diseases society of America/American thoracic society consensus guidelines on the management of community-acquired pneumonia in adults. Clin Infect Dis 2007;44:\$27-72. http://dx.doi.org/10.1086/511159

10. Furera V, Ravehb D, Picardc E, Goldbergc S, Izbickic G. Absence of leukocytosis in bacteraemic pneumococcal pneumonia. Prim Care Respir J 2011;20(3):276-81. http://dx.doi.org/10.4104/pcrj.2011.00023

11. Bartolomè M, Almirall J, Morera J, et al. and the Maresme Community-Acquired Pneumonia Study Group (GEMPAC). A population-based study of the costs of care for community-acquired pneumonia. Eur Respir J 2004;23:610-16. http://dx.doi.org/10.1183/09031936.04.00076704

12. Lim WS, Macfarlane JT, Levy ML, on behalf of the British Thoracic Society Community Acquired Pneumonia Guidelines Committee. Community acquired pneumonia guidelines for primary care - pragmatic management at the front line. BMJ 2010. http://www.bmj.com/content/341/bmj.c2916?tab=responses, last access: 09/11/2013

13. Mandell LA, Marrie TJ, Grossman RF, et al. Canadian guidelines for the initial management of community-acquired pneumonia: an evidence-based update by 
the Canadian Infectious Diseases Society and the Canadian Thoracic Society. Clin Infect Dis 2000;31:383-421. http://dx.doi.org/10.1086/313959

14. Menéndez R, Torres A, Aspa J, Capelastegui A, Prat C, Rodríguez de Castro F. Community-Acquired Pneumonia. New Guidelines of the Spanish Society of Pulmonology and Thoracic Surgery (SEPAR). Arch Bronconeumol
2010;46(10):543-58. http://dx.doi.org/10.1016/j.arbres.2010.06.014

15. Woodhead M, Blasi F, Ewig S, et al. Joint Taskforce of the European Respiratory Society and European Society for Clinical Microbiology and Infectious Diseases. Guidelines for the management of adult lower respiratory tract infections. Clinical Microbiology and Infection 2011;17:(Suppl 6)

\section{Correct inhalation technique is critical in achieving good asthma control}

\author{
*Federico Lavorini', Omar S Usmani² \\ 1 Careggi University Hospital, Department of Experimental and \\ Clinical Medicine, Florence, Italy \\ 2 National Heart and Lung Institute, Imperial College London \& \\ Royal Brompton Hospital, London, UK \\ *Correspondence: Dr Federico Lavorini, Careggi University \\ Hospital, Department of Experimental and Clinical Medicine, \\ Largo Brambilla 3, 50134, Florence, Italy \\ Tel: +39-055-7947516 Fax: +39-055-422320 \\ E-mail: federico.lavorini@unifi.it
}

See linked article by Levy et al. on pg 406

The prevalence of asthma has been reported to range from 1 to $18 \%$ of the population in different countries. ${ }^{1}$ This means that several hundred million patients with asthma rely on the efficacy of their inhalers to achieve asthma control. Undeniably, inhaled therapy remains the cornerstone of treatment for patients with asthma, and the main inhalation devices used are pressurised metered dose inhalers (pMDIs) and dry powder inhalers (DPIs). ${ }^{2}$ Yet there are more than 200 different drug-inhaler combinations available in any medical formulary, and this causes immense confusion amongst prescribers, healthcare professionals and patients.

Published evidence shows that, when used correctly, there is little difference in clinical efficacy between different inhaler types. ${ }^{3}$ However, several studies have reported that a high proportion of patients cannot use their inhalers (either pMDIs or DPIs) well enough to benefit from the treatment. ${ }^{4,5}$ These numbers are even more depressing considering that between 40 to $85 \%$ of healthcare professionals, who should readily be able to teach patients how to use their inhalers correctly, do not seem to be able to perform that task properly - and doctors are the worst amongst all healthcare professionals. $^{6-8}$

It has clearly been shown that failure to use inhalers correctly may result in poor asthma control, ${ }^{8}$ increased cost, ${ }^{9}$ and a greater risk to the patient from exposure to less well-tolerated alternative treatments. In a large cross-sectional study involving over 1600 asthma outpatients, the finding of just one critical error in inhalation technique, irrespective of the inhalation device (DPI or pMDI), was associated with increased emergency room visits, hospitalisation and oral medication prescription. ${ }^{10}$

In this issue of the $P C R J$, Levy and co-workers ${ }^{11}$ retrospectively evaluated pMDIs usage in a large cohort (nearly 4000) of UK primary care patients with asthma, and correlated patients' inhaler technique with the level of asthma control. Patients at British Thoracic Society (BTS) treatment steps 1, 2 and 3 had their asthma status reviewed through the Improving the Management of Patients Asthma and COPD Treatment (IMPACT) service, where specialist nurse advisors undertake asthma reviews in primary care according to protocols based on the UK BTS/SIGN National Asthma Guidelines. ${ }^{12}$ Interestingly, part of the review involved evaluating $\mathrm{pMDI}$ inhaler technique objectively by using the Vitalograph Aerosol Inhalation Monitor, ${ }^{13}$ a training device aimed at assessing three crucial steps needed for correct pMDI usage: slow inhalation flow (below $50 \mathrm{~L} / \mathrm{min}$ ); synchronisation between inhaler actuation and inhalation; and a 5 second breath-hold pause following inhalation.

The authors observed that patients who displayed significant errors when using pMDIs had higher risks of poor asthma control and more bursts of systemic corticosteroid prescriptions than those who operated pMDIs correctly. ${ }^{11}$ Of note, patients who were using pMDIs in conjunction with spacers, or were using breath-actuated inhalers, had better asthma control than those using pMDIs alone. Synchronisation - i.e. achieving the correct inhalation flow following actuation - was the main step in the inhalation technique which most patients failed.

These findings should be interpreted in the context of the retrospective observational nature of the study. We do not know whether other reasons for poor disease control (e.g. co-morbidities, different treatment plans, different drug dosages) were more frequent in patients who misused their pMDI than those who used it correctly. In addition, the UK is rather atypical with respect to device prescription compared to the rest of Europe, where DPIs are the favoured inhalation device. In this current study, only $9 \%$ and $14 \%$ of patients, respectively, used their DPI as reliever, or maintenance, therapy. The investigators did not attempt to assess inhalation technique in the patients prescribed a DPI, nor did they relate DPI technique to asthma control. Certainly there is evidence to show that patients using DPIs may experience more critical errors than those using pMDls. ${ }^{10}$ Despite these limitations, the results of the study by Levy et al. are important: they confirm the relationship between inhaler misuse and poor asthma control, and reinforce the notion of the importance of patients training for efficient inhalation drug delivery. ${ }^{11}$

Patients' ability to handle inhalers correctly is a crucial issue for the choice of the most appropriate inhaler device for a given patient. Adherence to therapy is likely to be influenced by patients' attitudes and their experience in using the device, and if the patient feels that 